\title{
Development of a Modified Naturalistic Action Test for Korean Patients With Impaired Cognition
}

\author{
Mi Ae Jung, MD, Hyun Jung Kim, MD, Youn Joo Kang, MD, Yon Joon Kim, MD
}

Department of Rehabilitation Medicine, Eulji General Hospital, Eulji University School of Medicine, Seoul, Korea

\begin{abstract}
Objective To develop and evaluate the psychometric properties of a modified Naturalistic Action Test (m-NAT) for Korean patients with impaired cognition. The NAT was originally designed to assess everyday action impairment associated with higher cortical dysfunction.

Methods We developed the m-NAT by adapting the NAT for the Korean cultural background. The m-NAT was modeled as closely as possible on the original version in terms of rules and scoring. Thirty patients receiving neurorehabilitation (twenty-three stroke patients, five traumatic brain injury patients, and two dementia patients) and twenty healthy matched controls were included. Inter-rater reliability was assessed between two raters. Validity was evaluated by comparing the m-NAT score with various measures of attention, executive functions, and daily life.

Results Performance on the m-NAT in terms of the total score was significantly different between patients and controls $(p<0.01)$. Patients made significantly more total errors than controls $(p<0.01)$. Omissions error was the most frequent type of error in patient group. Intraclass correlation coefficients for total m-NAT score was 0.95 (95\% confidence interval [CI], 0.92 to $0.97 ; \mathrm{p}<0.001)$; total error was 0.91 (95\% CI, 0.89 to $0.92 ; \mathrm{p}<0.001)$. Total m-NAT score showed moderate to strong correlations with Stroop test interference score \& index, Trail Making Test parts A and B, Sustained Attention to Response Task commission error, Functional Independence Measure, Korean instrumental activities of daily living, Korean version of the Cognitive Failures Questionnaire, and Executive Behavior Scale $(\mathrm{p}<0.05)$.

Conclusion The m-NAT showed very good inter-rater reliability and adequate validity. The m-NAT adjusted to Korean cultural background can be useful in performance-based assessment of naturalistic action for clinical and research purposes.
\end{abstract}

Keywords Cognition, Executive function, Rehabilitation

Received April 4, 2012; Accepted June 26, 2012

Corresponding author: Hyun Jung Kim

Department of Rehabilitation Medicine, Eulji General Hospital, 68 Hangeulbiseok-ro, Nowon-gu, Seoul 139-711, Korea

Tel: +82-2-970-8316, Fax: +82-2-972-0068, E-mail: khj2603@eulji.ac.kr

(c) This is an open-access article distributed under the terms of the Creative Commons Attribution Non-Commercial License (http://creativecommons. org/licenses/by-nc/3.0) which permits unrestricted noncommercial use, distribution, and reproduction in any medium, provided the original work is properly cited.

Copyright $\odot 2013$ by Korean Academy of Rehabilitation Medicine

\section{INTRODUCTION}

Brain lesions can lead to deficits in single domains of cognitive function, such as memory, learning, attention, visuospatial perception, or language, but they can also lead to more complex deficits in higher-order cognitive ability i.e., executive function [1]. These cognitive deficits can have a negative impact on a patient's motivation for 
rehabilitation and on the learning process required for regaining motor skills, which in turn may lead to further functional decline and a delay in their return to society.

Traditionally, the clinical consequences of frontal lobe damage, such as a difficulty in maintaining goal-directed behavior, impulsivity, emotional labiality, and apathy are collectively referred to as "frontal lobe syndrome" or executive dysfunction [2,3]. However, according to Goldberg and Bougakov [4], the executive dysfunction is not only restricted due to frontal lobe damage, but also appeared in various areas as a result of the anatomical complexity of the brain. Therefore, a more functional approach is required for sufficient assessment of executive function.

Executive functions are integrative, higher level, cognitive processes that control and regulate other cognitive processes in order to set up strategy and to generate and maintain goal-directed behavior according to everyday life priorities $[5,6]$. In other words, it is a comprehensive term used to describe a variety of processes, such as initiation, organization and planning, hypothesis formulation, making decisions, self-monitoring and adjustment, and utilizing feedback [5,6]. Executive function also encompasses a person's ability to control their behavior, thus enabling them to be socially competent, while executive dysfunction can lead to adverse behavior, such as outbursts of emotion or declining motivation. This in turn can interfere with their social and occupational roles and impair their quality of life [6]. Patients with stroke, dementia, and traumatic brain injury frequently experience executive dysfunction. Therefore, proper assessment of executive function is of particular importance in the field of neurorehabilitation, especially when it comes to the planning of appropriate treatment programs. However, due to the difficulties in conceptualization of executive function, a clinically valid test for assessing patients with executive dysfunctions has not been developed enough.

Conventional tests of executive function, like the Wisconsin Card Sorting Test were not originally developed to assess deficits in real-world activities [7]. Several studies criticize these conventional tests as lacking sensitivity to identify everyday life problems that can occur in patients with clinically significant executive dysfunction, and thus highlight the potential invalidity of using these tools [79]. This criticism arises from the fact that conventional laboratory-based tests are designed to assess specific individual components of executive function while the patient is in a relatively well-supported and structured environment, far different from community settings [79]. In daily life, individuals encounter more complicated, novel, and challenging situations that require the active participation of both goal-directed and multi-tasking abilities.

For this reason, the benefits of assessing problem-solving abilities and performances in an open-ended naturalistic setting that reflects the real-world functioning of the individual has been emphasized [7,9]. Recently, there have been many attempts to develop such tests that can better identify everyday life difficulties that can arise in patients with executive dysfunction. For example, the Multiple Errands Test (MET), originally developed by Shallice and Burgess [10], requires participants to multitask efficiently in a shopping precinct. Additionally, a simplified version of the MET for a hospital setting has been developed, as well as site-specific versions for different hospitals $[11,12]$. The Multi-Level Action Test (MLAT) is a laboratory-based test that assesses an individual's ability to multitask [13]. However, due to the lack of standardization, its time-consuming nature, and the difficulty in scoring the MLAT, Schwartz et al. [14] developed the Naturalistic Action Test (NAT) by reducing the test time and simplifying the scoring of the MLAT. The NAT is a standardized, performance-based measure that assesses naturalistic action impairments that occur in everyday activities due to higher cortical dysfunction. The NAT discriminates well between participants with acquired brain injury (i.e., traumatic brain injury and stroke) and their matched controls, with adequate reliability and validity, and thus emphasizes the appropriateness of its use as a screening tool [14].

However, in Korea, there is currently no test available to evaluate executive function in a real-world setting, despite the above-mentioned critical clinical implications. The Kims Frontal-Executive Neuropsychological Test (K-FENT) [15] and subtest of frontal-executive functions in the Seoul Neuropsychological Screening Battery are frequently used to assess executive function in a clinical setting. However, these tests are a collection of traditional measures of executive function.

Thus, an assessment tool that can reflect and characterize an individual's performance of executive dysfunc- 
tion in the everyday world is needed. In this study, we developed the modified Naturalistic Action Test (m-NAT) by adapting the NAT test for the Korean cultural background. In addition, we evaluated the psychometric properties of the m-NAT in Korean patients with impaired cognition.

\section{MATERIALS AND METHODS}

\section{Participants}

Thirty participants who had been diagnosed with stroke $(\mathrm{n}=23)$, traumatic brain injury $(\mathrm{n}=5)$, vascular dementia $(n=1)$, or Alzheimer's dementia $(n=1)$ were recruited into this study. All participants were patients using the neurorehabilitation service of Eulji General Hospital between May 2010 and August 2011, and all were neurologically and medically stable. Their disease duration was diverse, with a minimal of 20 days and a maximal of 20 years. They were comprised of 14 men and 16 women, with a mean age of $64.9 \pm 9.4$ years and mean education duration of $8.5 \pm 3.3$ years. Subjects were excluded if they were unable to follow and or perform tasks due to 1) any auditory and/or visual dysfunction, 2) insufficient sensorimotor capacity, 3) incompetent to understand and follow task instructions, 4) unable to participate attentively and control behavior appropriately at least for 1 hour testing session, or 5) Beck's depression score more than 25.

The control group consisted of 20 healthy subjects without any history of neurological or psychiatric conditions, and had a Korean Mini-Mental State Examination score above 26. They consisted of 9 men and 11 women with a mean age of $63.9 \pm 6.9$ years and mean education duration of 8.9 \pm 3.4 years. There were no significant differences with regards to age, gender, and duration of education between the two groups.

This study was approved by the Institutional Review Boards of Eulji General Hospital, and each participant gave informed written consent.

Neuropsychological tests and measures of everyday functioning

Patients were first evaluated using a battery of neuropsychological tests and measures of everyday functioning. Attention and executive functions were measured using the Stroop test [15], the Trail Making Test parts A and B, and the Sustained Attention to Response Task (SART)
[16]. Daily functioning was measured using the Functional Independence Measure (FIM) [17], the Korean instrumental activities of daily living (K-IADL) [18], the Korean version of the Cognitive Failures Questionnaire (K-CFQ) [19], and the Executive Behavior Scale (EBS) [15]. The $\mathrm{K}-\mathrm{CFQ}$ is a self-administered questionnaire, designed to measure lapses in cognition during daily life. The EBS is a questionnaire included in K-FENT, completed by a significant other of the patient and focused on measuring personality/emotional changes and behavioral problems associated with dysexecutive syndrome. A well-trained, experienced examiner conducted all of the evaluations.

\section{Modified Naturalistic Action Test \\ Test development}

The authors obtained permission from the original developer to use and adapt the NAT. A detailed instruction manual and scoring guide for the NAT can be downloaded from the Website address, http://www.mrri.org/ naturalistic-action-test.

The NAT, precursor to the m-NAT, used three primary tasks: 1) make toast with butter and jam and instant coffee with cream and sugar; 2) wrap a gift (i.e., doll) as a present; 3) prepare and pack a child's lunchbox with a sandwich, snack and a drink, and pack a child's school bag. At first, the second task "wrap a gift as a present" was modified to the task "fold the laundry and put it in a dresser," because the original task "wrap the doll" was revealed as culturally unfamiliar to Korean elderly people in our preliminary study. However, the modified task "fold the laundry and put it in a dresser" failed to discriminate between the patient and the control groups. Therefore, in the end, the m-NAT was simplified by using 2 tasks primarily based on the original version, whereby task 3 of the original NAT corresponded to task 2 of the m-NAT. The third task of the original NAT is comprised of 10 steps. Amongst these steps, the original "make a sandwich" was modified to "prepare a lunchbox with rice," while "wrap sandwich with foil" was modified to "prepare a lunchbox with side dish." In addition, the target objects of a bottle of juice and a binder were substituted with a bottle of water and a book.

Completed steps for each task were expressed as a proportion, and the score related to the accomplishment score. Every instance of omission and commission error was recorded in detail. The m-NAT score, a six-point 
scale for each task, was derived from a combination of accomplishment and error scores for each task, according to the scoring rule.

\section{Procedure}

m-NAT was administered to all participants, the participant was required to perform the m-NAT while seated at a U-shaped table. All materials were placed uniformly on a table within reach at the start of each task. Because the NAT is designed not to penalize for physical limitations, such as weakness and range of motion limitations, the examiner was permitted to give physical assistance when subject requested help, but only to fulfill a step that the subject had already initiated and with strict restrictions. While explaining the task, the examiner showed pictures of the completed task. Subjects were asked to repeat the task instructions before each task began and were permitted to start only if they showed a thorough understanding of the task. If the subject had aphasia, the examiner needed to confirm that the instructions had been understood by using the gestures, writing or answer to simple yes/no questions. During the assessment, the examiner avoided eye contact and kept conversation to a minimum with participants. The maximum time allowed to complete each task was 30 minutes, and the performance of each subject was videotaped. One rater administered the m-NAT to the subjects using a written script, and they scored the tasks while there were being carried out. Another rater independently scored the tasks, using the videotape of the m-NAT test to measure the interrater reliability.

\section{Statistical analysis}

Statistical analysis used the SPSS ver. 12.0 (SPSS Inc., Chicago, IL, USA). Between-group differences in performance, in terms of their accomplishment score, error score, and m-NAT score for each task were examined with independent sample t-tests. To measure validity, the correlation between the total m-NAT score and the scores from the pre-evaluation tests in the patient group was compared using Spearman's correlation coefficients. Regarding the total m-NAT score, gender differences were also examined using independent sample t-tests. In addition, Spearman's correlation test was performed to confirm whether age and/or education duration influenced the total m-NAT results. The inter-rater reliability was evaluated using intraclass correlation coefficient (ICC), with $95 \%$ confidence interval (CI).

\section{RESULTS}

\section{m-NAT performance}

Performance of the m-NAT is shown in Table 1. For task 1 , the accomplishment score was significantly impaired in the patient group $(\mathrm{p}<0.05)$. For task 2 , the patient group performed significantly worse than the controls in terms of the accomplishment score and made significantly more errors $(\mathrm{p}<0.001)$. When the accomplishment and error scores for each task were combined into the m-NAT score, performances of the patients were significantly more impaired than the controls for both tasks $(\mathrm{p}<0.001)$. The mean duration of time to complete the m-NAT was significantly longer for the patient group $(\mathrm{p}<0.05)$.

The mean total number of errors in the patient group was 6.9 , and this was significantly higher than that of the control group $(3.4, \mathrm{p}<0.001)$. An error analysis was conducted in detail as it was done by Schwartz et al. [13]. The m-NAT errors, categorized as omission, sequence, substitution, gesture, spatial estimation, tool omission, action addition, and quality are displayed in Table 2 . Omission error was rare in the control group, whereas errors in this category were more than 4 times higher in the patient group. The following are examples of omissions

Table 1. Modified Naturalistic Action Test (m-NAT) performances in patient and control groups

\begin{tabular}{lccr}
\hline & $\begin{array}{c}\text { Patient } \\
\text { group (n=30) }\end{array}$ & $\begin{array}{c}\text { Control } \\
\text { group (n=20) }\end{array}$ & \multicolumn{1}{c}{$\begin{array}{c}\text { p- } \\
\text { value }\end{array}$} \\
\hline $\begin{array}{l}\text { Accomplishment } \\
\text { score (\%) }\end{array}$ & & & \\
Task 1 & $86.0 \pm 16.4$ & $94.1 \pm 9.9$ & 0.035 \\
\hline Task 2 & $55.3 \pm 22.1$ & $85.0 \pm 14.3$ & $<0.001$ \\
\hline Total error (no.) & $6.9 \pm 1.8$ & $3.4 \pm 2.6$ & $<0.001$ \\
\hline Task 1 & $1.7 \pm 0.7$ & $1.4 \pm 0.9$ & 0.109 \\
\hline Task 2 & $5.2 \pm 1.0$ & $2.0 \pm 1.6$ & $<0.001$ \\
Total m-NAT score & $5.2 \pm 2.6$ & $9.0 \pm 2.2$ & $<0.001$ \\
\hline Task 1 (out of 6) & $3.9 \pm 1.6$ & $5.1 \pm 1.2$ & 0.003 \\
\hline Task 2 (out of 6) & $1.3 \pm 1.3$ & $3.9 \pm 1.8$ & $<0.001$ \\
Time to completion & $26.7 \pm 8.7$ & $18.5 \pm 5.3$ & 0.012 \\
(min) & & & \\
\hline
\end{tabular}

Values are presented as mean \pm standard deviation. $\mathrm{p}$ values were obtained by independent sample t-tests. 
Table 2. Error frequencies by groups

\begin{tabular}{lcc}
\hline \multicolumn{1}{c}{ Error } & $\begin{array}{c}\text { Patient group } \\
(\mathbf{n}=\mathbf{3 0})\end{array}$ & $\begin{array}{c}\text { Control group } \\
(\mathbf{n = 2 0})\end{array}$ \\
\hline Omission & 0.38 & 0.09 \\
Sequence & 0.17 & 0.14 \\
\hline Substitution & 0.09 & 0.02 \\
Gesture & 0.02 & - \\
\hline Spatial estimation & 0.02 & - \\
\hline Tool omission & 0.02 & 0.01 \\
\hline Action addition & 0.01 & - \\
\hline Quality & 0.03 & 0.01 \\
\hline
\end{tabular}

Values are presented as proportions of all errors observed in both groups. Total number of errors for the patient group was 185, and for the control group was 64 .

by patients: toast - not apply butter or jam; coffee - not add coffee powder, sugar, or cream; lunchbox - not add side dish; and schoolbag - not pack markers. When we excluded the omissions and looked solely at the commission errors, the proportional distribution of commission errors categorized for both groups proved to be roughly similar. However, the commission errors recorded for the patient group had a more distinct pattern than that of the control group. The following are examples of flagrant errors by patients: substitution - spread sugar or coffee on toast; sequence - apply butter continuously; gesture place lid with press rather than screw motion.

For all participants, we found no association between education duration and m-NAT scores for each task as well as total m-NAT score. There was no significant gender difference on m-NAT scores for each task and overall total m-NAT score. Age showed a significant negative correlation with total m-NAT score $(r=-0.329, p<0.05)$.

Validity: comparison of the m-NAT with various measures of attention, executive functions, and daily life

Thirty patients were evaluated for various measures of executive function, attention, and daily life (Table 3). The total m-NAT scores are statistically significantly correlated with all pre-evaluated test scores (Table 4, Fig. 1, 2).

\section{Inter-rater reliability}

To determine inter-rater reliability, ICCs were evaluated on the m-NAT measurements for each task, including number of errors, accomplishment score, and m-NAT score. ICCs for the number of errors for task 1 was 0.86
Table 3. Performance of the patient group on the neuropsychological tests and measures of everyday functioning

\begin{tabular}{|cc}
\hline \multicolumn{1}{c}{ Measure } & Patient group $(\mathbf{n = 3 0})$ \\
\hline Stroop test & \\
\hline Interference score $(\mathrm{sec})$ & $24.9 \pm 19.3$ \\
\hline Interference index $(\%)$ & $21.1 \pm 25.0$ \\
\hline Trail Making Test-A (sec) & $71.2 \pm 35.9$ \\
\hline Trail Making Test-B (sec) & $115.7 \pm 71.8$ \\
\hline SART commission error & $18.8 \pm 6.6$ \\
\hline FIM total (18-126) & $88.4 \pm 19.6$ \\
\hline FIM motor (13-91) & $66.5 \pm 16.2$ \\
\hline FIM cognitive (5-35) & $20.9 \pm 6.02$ \\
\hline K-IADL (0-33) & $17.5 \pm 7.3$ \\
\hline K-CFQ (0-100) & $55.5 \pm 21.9$ \\
\hline EBS $(0-100)$ & $53.9 \pm 20.5$ \\
\hline
\end{tabular}

Values are presented as mean \pm standard deviation. SART, Sustained Attention to Response Task; FIM, Functional Independence Measure; K-IADL, Korean Instrumental Activities of Daily Living; K-CFQ, Korean version of the Cognitive Failures Questionnaire; EBS, Executive Behavior Scale.

Table 4. Correlation between total m-NAT score and neuropsychological tests and measures of everyday functioning

\begin{tabular}{|lc|}
\hline \multicolumn{1}{c}{ Measure } & Total m-NAT score \\
\hline Stroop test & $-0.562^{* *}$ \\
\hline Interference score $(\mathrm{sec})$ & $0.649^{* * *}$ \\
\hline Interference index $(\%)$ & $-0.562^{* *}$ \\
\hline Trail making test-A $(\mathrm{sec})$ & $-0.733^{* * *}$ \\
\hline Trail making test-B $(\mathrm{sec})$ & $-0.437^{* *}$ \\
\hline SART commission error & $0.563^{*}$ \\
\hline FIM total & $0.381^{*}$ \\
\hline FIM motor & $0.484^{* *}$ \\
\hline FIM cognitive & $-0.577^{*}$ \\
\hline K-IADL & $-0.506^{* *}$ \\
\hline K-CFQ & $-0.597^{* *}$ \\
\hline EBS
\end{tabular}

${ }^{*} \mathrm{p}<0.05,{ }^{* *} \mathrm{p}<0.01,{ }^{* * *} \mathrm{p}<0.001$ by Spearman's correlation test.

m-NAT, modified Naturalistic Action Test; SART, Sustained Attention to Response Task; FIM, Functional Independence Measure; K-IADL, Korean Instrumental Activities of Daily Living; K-CFQ, Korean version of the Cognitive Failures Questionnaire; EBS, Executive Behavior Scale. 
(A)

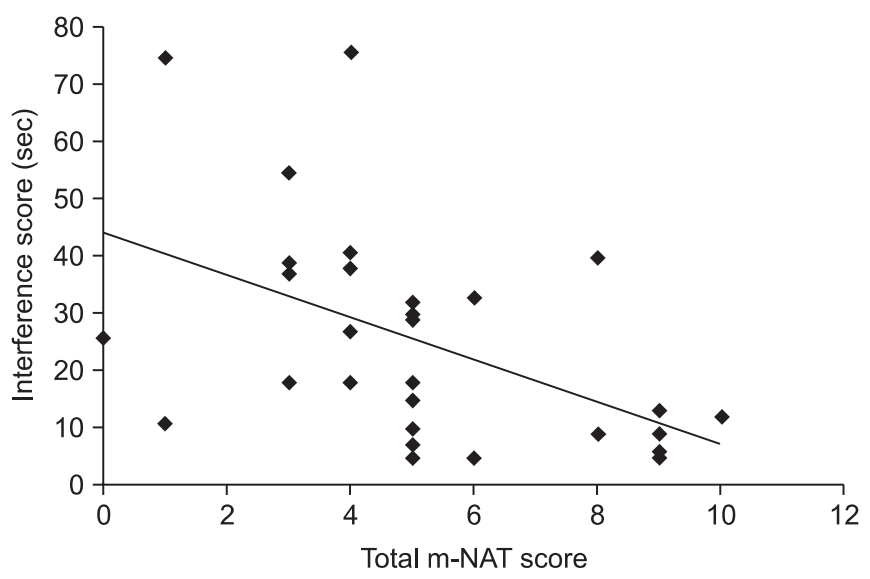

(B)

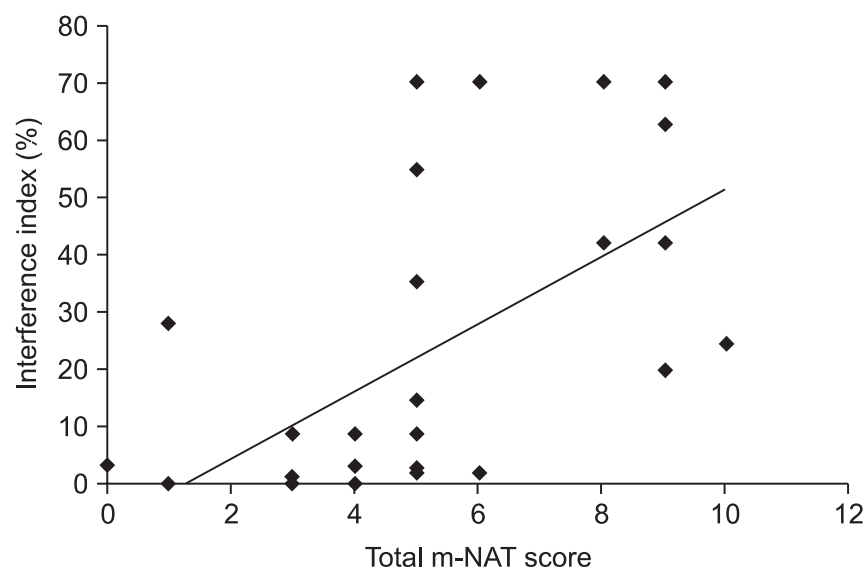

Fig. 1. (A) Correlation between interference score of Stroop test and total modified Naturalistic Action Test (m-NAT) score $(\mathrm{r}=-0.562, \mathrm{p}=0.001)$. (B) Correlation between interference index of Stoop test and total $\mathrm{m}$-NAT score $(\mathrm{r}=0.649$, $\mathrm{p}<0.001)$.

(A)

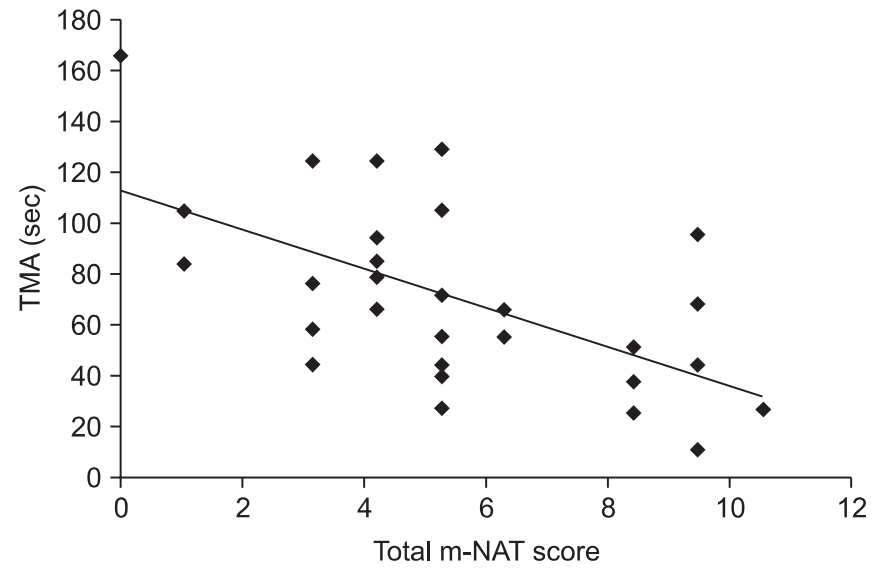

(B)

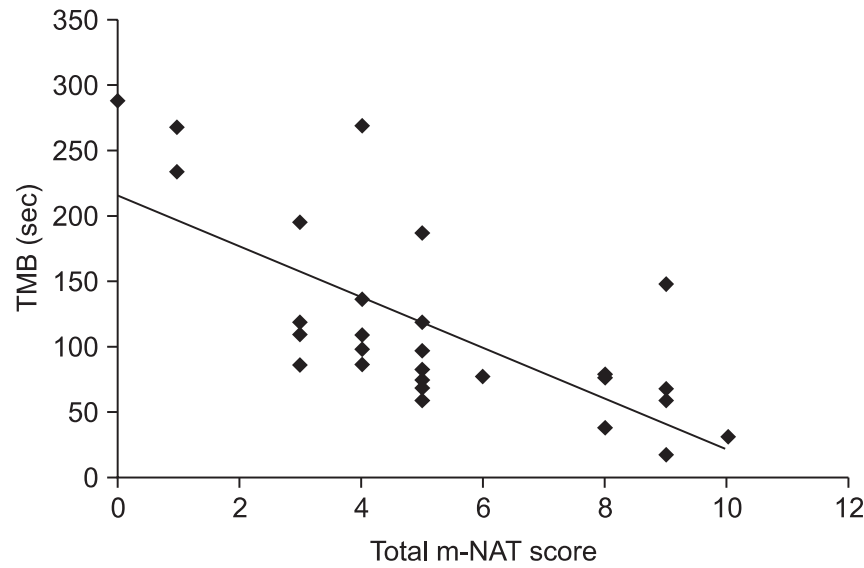

Fig. 2. (A) Correlation between Trail Making Test A (TMA) and total modified Naturalistic Action Test (m-NAT) score $(\mathrm{r}=-0.562, \mathrm{p}=0.001)$. (B) Correlation between Trail Making Test B (TMB) and total m-NAT score $(\mathrm{r}=-0.733, \mathrm{p}<0.001)$.

( $95 \%$ CI, 0.77 to 0.91 ) and for task 2 was 0.96 (95\% CI, 0.94 to 0.98 ). ICCs of the accomplishment score for task 1 was 0.86 (95\% CI, 0.77 to 0.92 ) and task 2 was 0.96 (95\% CI, 0.94 to 0.98 ). ICCs of the m-NAT score for task 1 was 0.85 (95\% CI, 0.75 to 0.91 ) and task 2 was 0.97 (95\% CI, 0.94 to 0.98 ). ICCs were all above 0.80 significance $(p<0.001)$, very good inter-rater reliability.

\section{DISCUSSION}

In this study, we developed the m-NAT in order to adapt the NAT for Korean cultural background; this modified test discriminated well between patients with cognitive impairment and controls. The validity of the m-NAT was supported by the demonstration of significant correlation between neuropsychological measures of executive function and attention tasks, and daily life assessments. Furthermore, ICCs were high, illustrating very good interrater reliability.

Our finding that the m-NAT has good discriminative ability coincides with findings from previous studies that have assessed executive function under naturalistic conditions $[10,11,14]$. In 1991, the MET was proposed as a prototype for the assessment of real-world executive function; in this study, three traumatic brain injury patients with relatively high-level functioning displayed 
disorganized performances in terms of rule breaks and inefficiencies when compared with controls [10]. The hospital version of the MET (MET-HV), developed thereafter, also discriminated well between patients and controls, with $85 \%$ sensitivity and $95 \%$ specificity [11]. Furthermore, the NAT developed by Schwartz et al. [14] displayed good validity and inter-rater reliability when conducted on 100 patients with acquired brain injury and 28 matched controls.

However, these tests were developed to suit Western culture and required transcultural modification in order to be appropriate for Korean culture and lifestyle. The first task derived from the original NAT, "make toast and instant coffee," was applied in exact accordance with the original script since this is a part of the general dietary habit in Korea. However, the third task of "prepare a lunchbox with a sandwich" was modified to "prepare a lunchbox with rice and side dishes" due to the differences in Western and Korean dietary habits. Additionally, the task of "fill thermos with juice" was modified to "fill thermos with water." Through these modifications, mNAT was simplified, but still displayed good discriminative ability. Moreover, the mean accomplishment score obtained from both groups was decreased in task 2 compared to task 1, thus demonstrating that the m-NAT possessed a gradation in task difficulty, like the original NAT.

In this study, the total number of errors committed by the patient group (mean, 6.9) was significantly higher than that of the control group (mean, 3.4). The number of omission errors was remarkably higher in the patient group when compared to controls; however, the commission error distribution was similar between groups. Of note, the commission errors in the patient group were more distinct and exaggerated than those observed in the control group. This finding may be partially explained by the theories of supervisory attentional system (SAS) and working memory model $[8,20,21]$. The SAS is involved in novel non-routine situations, and enables a subject to execute proper actions while suppressing improper actions, according to the priority of the actions [20,21]. The error frequency in our study corresponded to the complexity of the task. In addition, errors may also occur when subjects have difficulty in executing target actions without any external clues. This is usually a result of damage to their working memory, which temporarily saves and uses information.
However, errors were also observed in the control group that had no history of neurological or psychiatric conditions. According to Schwartz et al. [13], such results can be explained by the hypothesis of limited-capacity commodity. Other related general constructs, such as effort, attention, and cerebral activation can also be invoked. Individual performance variations in information processing and language tasks have been attributable in previous studies to the individual differences in available resource capacity $[13,22]$. Hence, a healthy person can still make errors because of their individual limited-capacity resources. Since the unique capacity resources of cognitive impaired patients is more restricted than that of controls, more significant and excessive errors can be observed. Of note, such errors tend to be omission errors when resource is severely restricted [13].

The construct validity of the m-NAT was examined against pre-evaluated neuropsychological tests. All measures of attention and executive functions demonstrated a significant correlation between accomplishment and m-NAT scores. The ecological validity of the m-NAT as a measure of deficits affecting functions in a real-world setting was supported by its significant correlation with measures of activities in daily living, such as FIM, KIADL, EBS, and K-CFQ. In addition, its correlations with the results from the Stroop test, SART commission error, and Trail Making Test indicate that the m-NAT is an effective index for the measurement of selective attention, inhibitory capacity, and cognitive flexibility aspects of executive function. The original NAT developed by Schwartz et al. [14], was statistically significantly correlated with FIM, Dual Task Test, Self-Ordered Pointing Test, and Star Cancellation Test, thus validating the NAT as a measure of functional disability, arousal, visuospatial attention, and working memory. The Baycrest Multiple Errands Test (BMET), standardized by Dawson et al. [12], is an ecologically valid measurement of cognitive function, supported by a good correlation with measures of IADL and daily life functioning.

Classifying the various types of errors committed by individuals while performing the m-NAT and analyzing the characteristics of the errors may help highlight deficits in the problem-solving capacity of patients with cognitive impairment, in terms of issues that may arise during their daily life. The discriminative ability and ecological validity of traditional executive function tests, such as the 
Wisconsin Card Sorting Test have recently been considered insufficient since they have little relevance to executive function in actual life [7,9]. Direct observation of the strategy used by patients under naturalistic performancebased assessments will not only assist in diagnosing problems at the impairment level, but will also provide valuable information to help clarify an individual's functionality at the activity and participation level [8]. Ultimately, this information can assist health care providers in designing and tailoring more effective rehabilitation interventions for individual patients with difficulties in everyday life functioning. Hence, it is necessary to carry out these tests in real life settings.

The m-NAT, like the original NAT, is an advantageous performance-based test in which the test environment, materials, and cueing procedures are all standardized. Physical assistance due to factors, such as limited movement capabilities of the patient and/or weakness does not affect the score. It is also a suitable test for subjects with aphasia, as long as they can demonstrate comprehension of the task instructions. Thus, in a broad sense, the m-NAT can be administered to any subjects presenting with brain injury.

The inter-rater reliability study demonstrated high agreement on all components. This finding replicates those of previous studies regarding the NAT and BMET $[12,14]$. In this study, the standardized instruction manual was translated into Korean to ensure the examiner provided the same instructions to all participants. Therefore, the m-NAT is suitable for use by any medical practitioners in a clinical setting once they have received a simple tutorial and have been acquainted with the instruction manual.

This study had some limitations. First, our participants were relatively old and their level of education was low. Controls were mostly recruited from caregivers or family members of the patients. They were matched as closely as possible with the patient group, regarding age, gender, and duration of education. The mean age of the all participants was over 60 years and the mean education duration was 8.7 years. There was no statistical difference between patient and control groups for the number of errors in task 1. Therefore, we cannot exclude the possibility that both groups, comprised mainly of elderly subjects, were unfamiliar with this task due to cultural aspects. Nevertheless, the tasks were configured to ensure that the difficulty level increased in a stepwise manner, similar to the tasks described by Schwartz et al. [14]. For this reason, we speculate that the errors obtained during the first task were not remarkable for both groups. More importantly, the accomplishment and the m-NAT scores revealed a statistically significant difference between the two groups. Accordingly, we suggest that first task had sufficient discriminative ability to differentiate between the patients and the control participants.

Another limitation of this study is that the pre-evaluation neuropsychological assessment could have been more comprehensive. We did not assess individual cognitive domains, such as language, visuospatial function, and memory, which may have affected our results.

Lastly, our sample size was small and made up of heterogeneous brain lesions. A further study with a larger sample size is required to discern any characteristic findings of the m-NAT with regard to specific pathologies or disease status.

In conclusion, the m-NAT, which was modified from the NAT in order to assess everyday action impairments in a Korean population, discriminated well between control individuals and patients with acquired brain lesions. The validity of the m-NAT was supported by the demonstration of significant correlation with various measures of attention, executive functions, and daily life. In addition, inter-rater reliability was good. Based on these results, we suggest that the m-NAT is a useful tool for investigating real-world performance difficulties that are related to executive dysfunction in Korean patients. Finally, further research is required to promote the m-NAT, recruiting a larger number of subjects with various backgrounds.

\section{CONFLICT OF INTEREST}

No potential conflict of interest relevant to this article was reported.

\section{ACKNOWLEDGMENTS}

We would like to thank Dr. Myrna F. Schwartz at Moss Rehabilitation Research Institute for generous permission to use and adapt the NAT for Korean populations. We would also like to thank Dr. John Whyte at Moss Rehabilitation Research Institute for his support. We would like to acknowledge that the Sustained Attention to Re- 
sponse Task was available to use in this study by Prof. Ian Robertson at Trinity College Dublin.

\section{REFERENCES}

1. Anderson CV, Bigler ED, Blatter DD. Frontal lobe lesions, diffuse damage, and neuropsychological functioning in traumatic brain-injured patients. J Clin Exp Neuropsychol 1995;17:900-8.

2. Stuss DT, Benson DF. Neuropsychological studies of the frontal lobes. Psychol Bull 1984;95:3-28.

3. Malloy P, Bihrle A, Duffy J, Cimino C. The orbitomedial frontal syndrome. Arch Clin Neuropsychol 1993;8:185-201.

4. Goldberg E, Bougakov D. Neuropsychologic assessment of frontal lobe dysfunction. Psychiatr Clin North Am 2005;28:567-80.

5. Fortin S, Godbout L, Braun CM. Cognitive structure of executive deficits in frontally lesioned head trauma patients performing activities of daily living. Cortex 2003;39:273-91.

6. Stuss DT, Levine B. Adult clinical neuropsychology: lessons from studies of the frontal lobes. Annu Rev Psychol 2002;53:401-33.

7. Burgess PW, Alderman N, Forbes C, Costello A, Coates LM, Dawson DR, et al. The case for the development and use of "ecologically valid" measures of executive function in experimental and clinical neuropsychology. J Int Neuropsychol Soc 2006;12:194-209.

8. Chan RC, Shum D, Toulopoulou T, Chen EY. Assessment of executive functions: review of instruments and identification of critical issues. Arch Clin Neuropsychol 2008;23:201-16.

9. Manchester D, Priestley N, Jackson H. The assessment of executive functions: coming out of the office. Brain Inj 2004;18:1067-81.

10. Shallice T, Burgess PW. Deficits in strategy application following frontal lobe damage in man. Brain 1991;114(Pt 2):727-41.

11. Knight C, Alderman N, Burgess PW. Development of a simplified version of the Multiple Errands Test for use in hospital settings. Neuropsychol Rehabil 2002;12:231-55.
12. Dawson DR, Anderson ND, Burgess P, Cooper E, Krpan KM, Stuss DT. Further development of the Multiple Errands Test: standardized scoring, reliability, and ecological validity for the Baycrest version. Arch Phys Med Rehabil 2009;90(11 Suppl):S41-51.

13. Schwartz MF, Montgomery MW, Buxbaum LJ, Lee SS, Carew TG, Coslett HB, et al. Naturalistic action impairment in closed head injury. Neuropsychology 1998;12:13-28.

14. Schwartz MF, Segal M, Veramonti T, Ferraro M, Buxbaum LJ. The Naturalistic Action Test: a standardised assessment for everyday action impairment. Neuropsychol Rehabil 2002;12:311-39.

15. Kim HK. Kims Frontal-Executive Neuropsychological Test. Daegu, Korea: Neuropsychology Press; 2001.

16. Robertson IH, Manly T, Andrade J, Baddeley BT, Yiend J. 'Oops!': performance correlates of everyday attentional failures in traumatic brain injured and normal subjects. Neuropsychologia 1997;35:747-58.

17. Uniform Data System for Medical Rehabilitation. Guide for use of the uniform data set for medical rehabilitation (adult FIM), version 4.0. Buffalo: State University of New York at Buffalo; 1993.

18. Kang SJ, Choi SH, Lee BH, Kwon JC, Na DL, Han S, et al. The reliability and validity of the Korean instrumental activities of daily living (K-IADL). J Korean Neurol Assoc 2002;20:8-14.

19. Kim HJ, Kang YJ, Park HK. Cross-cultural adaptation and development of Korean version of Cognitive Failure Questionnaire. Ann Bull Bum-Suk Acad Sch Found 2011;14:77-88.

20. Shallice T, Burgess P. Higher-order cognitive impairments and frontal lobe lesions. In: Levin HS, Eisenberg HM, Benton AL, editors. Frontal lobe function and dysfunction. New York: Oxford University Press; 1991. p. 125-38.

21. Norman DA, Shallice T. Attention to action: willed and automatic control of behavior. In: Gazzaniga MS, editor. Cognitive neuroscience: a reader. Malden, MA: Blackwell; 2000. p. 376-90.

22. Deneman M, Carpenter PA. Individual differences in working memory and reading. J Verbal Learning Verbal Behav 1980;19:450-66. 